\title{
COMPORTAMIENTO DE LA MADERA DE Pinus taeda IMPREGNADO CON ARSENIATO DE COBRE CROMATADO (CCA)
}

\author{
Teresa María Suirezs * \\ * Eng ${ }^{\text {a }}$ Florestal, M.Sc., Facultad de Ciencias Forestales, UnaM, Eldorado, Misiones, Argentina - suirezs@facfor.unam.edu.ar
}

Recebido para publicação: 13/09/2004 - Aceito para publicação: 14/02/2005

\begin{abstract}
Resumen
Comportamiento de la madera de Pinus taeda impregnado con Arseniato de Cobre Cromatado $(C C A)$. Este trabajo tuvo por objetivo, estudiar el comportamiento de las propiedades físicas y mecánicas de la madera de Pinus taeda L. impregnada por vacío-presión con preservador CCA (CrO3; CuO; As2O5) con tres retenciones, 5,10 y $15 \mathrm{~kg} / \mathrm{m}^{3}$. El proceso de impregnado se realizó, por el método Burnett, aplicándose presión y vacío de $7 \mathrm{~kg} / \mathrm{m}^{2}$ y $-0,5 \mathrm{~kg} / \mathrm{m}^{2}$ respectivamente. Los ensayos de las propiedades físicas y mecánicas se determinaron según lo establecen, las Normas técnicas IRAM (Instituto Argentino de Racionalización de Materiales), ASTM (American Society for Testing and Materials) y DIN (Deutsche Industrie Norm). Las propiedades físicas como ser los pesos específicos aparentes no son afectadas por las retenciones de CCA en la madera. Las contracciones tanto en el sentido tangencial como radial en las maderas impregnadas son menores. Las propiedades mecánicas de resistencia a la flexión estática, compresión paralela a las fibras, tracción perpendicular a las fibras, disminuyen levemente sus valores promedios con respecto a la madera sin impregnar, pero estas diferencias no son estadísticamente significativas, para $95 \%$ de confianza. La impregnación ha producido un efecto positivo en la dureza Janka transversal y en el corte paralelo a las fibras tangencial siendo estas diferencias estadísticamente significativas.
\end{abstract}

Palabras clave: Pinus taeda; impregnación con CCA; retenciones; propiedades físico-mecánicas.

\begin{abstract}
Summary
Behaviour of the wood of Pinus taeda impregnated with Chrome, Copper, Arsenic (CCA). The physical and mechanical properties of the wood of Pinus taeda L. without impregnating and impregnating by empty - pressure with preserving CCA (Chrome, Copper, Arsenic) with three retentions, 5, 10 and $15 \mathrm{~kg} / \mathrm{m}^{3}$ have been determined and analysed. The impregnation was accomplished in an autoclave applying the Burnett method. The physical and mechanical properties were determined according to the following technical Procedures; IRAM (Argentine Institute for Rationalization of materials), ASTM (American Society for Testing and Material) and DIN (Deutsche Industrie Norm). The results indicate that the specific weights of samples containing different percentages of humidity was not affected by the retentions of CCA in the wood. The shrinkage both in the tangential and radial directions in the impregnated samples were smaller in the impregnated sample. The mechanical resistance to statics flexion, compression parallel to the fibers, traction perpendicular to the fibers, hardness tangential and radial Janka and paralell radial cut, do not show statistically meaningful differences between impregnated and not impregnated samples; however the treatment has produced a positive effect in the hardness transverse Janka and in the parallel cut to the tangential fibers.
\end{abstract}

Keywords: Pinus taeda; impregnation with CCA; retentions; physical and mechanical properties.

\section{INTRODUCCIÓN}

Las coníferas tienen importancia como maderas aserradas para la construcción, por su estabilidad estructural y por su resistencia a la rajadura al ser clavadas. En muchas partes del mundo son demandadas para molduras, marcos de ventanas, puertas (SAGPyA. 1999).

Se ha demostrado que muchas coníferas implantadas de rápido crecimiento presentan buenas resistencias mecánicas, las que la hacen aptas para varios usos y también hay que destacar la resistencia en relación a su peso, ya que es una madera medianamente liviana. González et al. (1992). 
La madera del Pinus taeda L. tiene escasa durabilidad natural, en condiciones de uso exterior y en contacto con el suelo. Se ha comprobado en otros países que la madera impregnada con CCA, alcanza una vida útil de aproximadamente 15 a 40 años en servicio, como ser torres de enfriamiento, muelles, postes, tutores (Wilkinson, 1979).

Según Pelagallo (1997), el uso de la madera impregnada está poco extendida en la Argentina. La proporción de la misma en la producción de pino no llega al 5\%.

Las maderas tratadas con productos químicos no deben ser cepilladas, perforadas, etc. con el fin de evitar cualquier tipo de contaminación ambiental; por ese motivo se recomienda realizar todas las terminaciones posibles antes de impregnarlas (Lepage, 1986).

La impregnación influye sobre el posterior procesamiento de la madera y su comportamiento. Uno de ellos es la medición de la humedad de la madera impregnada, ya que la medición con xilohigrómetro se basa en la relación entre la humedad de la madera y sus propiedades eléctricas. El preservante CCA afecta las propiedades eléctricas de la madera, ya que las lecturas de la humedad dan valores superiores a los reales (Ramirez, 1996).

Según Tinto (1980), las maderas tratadas con preservantes hidrosolubles no presentan inconvenientes en la aplicación de pinturas, barnices y lustres siempre que el material esté seco. La incorporación de productos preservantes en la masa leñosa afecta a sus propiedades en sentido positivo, dándole mayor resistencia frente al ataque de los agentes destructores, pero a veces aparecen reacciones negativas, como ser la reducción de sus resistencias mecánicas, porque la madera se vuelve quebradiza; esto depende del tipo de tratamiento y la concentración del producto.

Las maderas tratadas con CCA son más duras, favoreciendo en este caso su uso como durmientes para ferrocarriles, como se ha demostrado en un estudio realizado en Nigeria sobre la instalación de durmientes impregnados con CCA. Después de haber estado 15 años en uso estos se encontraban en perfectas condiciones (Wilkinson, 1979).

Pizzi et al, citado por Lepage (1986), afirma, la madera impregnada con CCA pierde resistencia a la tenacidad. Esta pérdida está relacionada positivamente con el aumento de la retención.

De acuerdo con Wilkinson (1979), la madera impregnada con CCA adquiere un color verde, sin embargo esta coloración puede atenuarse estacionando la misma bajo cubierta hasta que se produzcan las reacciones químicas del producto con la madera. También se puede agregar al preservante pigmentos con el fin de mantener el color natural de la madera.

Una vez impregnadas las maderas, estas deben estacionarse como mínimo 7 días a $25^{\circ} \mathrm{C}$ para que se produzcan todas las reacciones químicas, y lograr la fijación del producto, por lo tanto deben estar protegidas de la lluvia para evitar que se diluya el producto que se encuentra en la superficie de la madera antes de su fijación, una vez seca y fijado el preservante dentro de la madera puede ser manipulada sin riesgo para el hombre y los animales domésticos (Wilkinson, 1979).

Un estudio realizado en el Reino Unido sobre maderas impregnadas con CCA en servicio por 11 años en torre de enfriamiento, no ha mostrado signo de lixiviación. (Wilkinson, 1979).

El CCA es un producto hidrosoluble que combina el efecto fungicida del cobre con el insecticida del arsénico y una sal alcalina de cromo con el fin de obtener una alta fijación de los principios activos de los componentes mencionados (Tuset y Duran, 1979).

Las maderas impregnadas con CCA son menos ignífugas que las maderas sin impregnar, pero una de sus características es, que una vez iniciado el fuego tiene la tendencia a continuarlo, por esa razón se le agregan aditivos ignífugos en su composición.

El CCA (Arseniato de cobre cromatado) se ha comenzado a usar desde 1930 y su aplicación ha sido creciente desde entonces. Su formulación ha cambiado. Se lo puede obtener en forma de sales cristalinas, pastas o líquidos concentrados (Fahlstrom, 1978).

Según A.W.P.A. (1996), establecen tres tipos de formulaciones distintas del CCA, clasificados según el porcentaje de óxidos en su composición, denominados A, B y C.

Esta clasificación fue establecida por AWPA después de varios experimentos realizado por Smith, D.N.R. y Williams, A.I. quiénes verificaron que la máxima eficiencia no coincide con la máxima fijación de cobre y arsénico (Lepage, 1986).

El objetivo de este trabajo fue evaluar el comportamiento de las propiedades físicas y mecánicas de la madera del Pinus taeda L. sin impregnar e impregnadas por vacío-presión con CCA y determinar si 
existe variación en las propiedades físicas y mecánicas de la madera impregnada con distintas retenciones de CCA.

\section{MATERIALES Y METODOS}

Para este trabajo, se seleccionaron al azar 6 árboles de Pinus taeda L. provenientes de una plantación comercial de 14 años de edad en un suelo rojo profundo, situado en el Municipio de Caraguatay, Departamento: Montecarlo, Misiones. Raleos a los 9 y 11 años. DAP medio de los árboles extraídos es fue de $31 \mathrm{~cm}$.

Se seccionaron toras desde la base hasta $2,40 \mathrm{~m}$, que fueron aserradas obteniéndose cuatro viguetas de cada una, a estas se la estacionaron bajo cubierta para su secado natural, luego se obtuvieron 2 probetas por árbol, por ensayo y por tratamiento, con 10 repeticiones. Este tamaño de muestra se debe a que las variaciones entre los árboles son mas significativas que la variación dentro del mismo árbol, de manera que una precisión mucho mayor con respecto a los valores de la media, se obtiene con mas árboles de diferentes sitios y menos muestras de cada árbol, para la determinación de las propiedades de la madera, es suficiente el rango de exactitud de la media del $15 \%$. Lo que significa, que solo se requieren 5 a 6 árboles seleccionados al azar. (Coronel, 1994)

Las probetas se realizaron según las Normas IRAM N 9544, 9543 y 9532, DIN $\mathrm{N}^{\circ} 52186$ y ASTM N $N^{\circ} 143 / 52$, luego fueron impregnadas en un autoclave para ensayos de laboratorio con el preservante $\mathrm{CCA}(\mathrm{CrO} 3$; $\mathrm{CuO}$; As2 $\mathrm{O} 5)$, tipo $\mathrm{C}$, con tres concentraciones distintas, tales que permitiera retenciones de 5,10 y $15 \mathrm{~kg} / \mathrm{m}^{3}$. Para todo los casos se aplicaron las mismas etapas y condiciones de impregnación: 1) vacío inicial hasta $-0,5 \mathrm{~kg} / \mathrm{cm}^{2}$ que se mantuvo por 30 minutos; 2) carga del autoclave con el producto preservante (CCA), aprovechando el vacío; 3) Presión de $7 \mathrm{~kg} / \mathrm{cm}^{2}$ durante 3 horas; 4) descarga del preservante; 5) extracción de las probetas, no se aplicó el vacío final con el fin de asegurarse la retención deseada. Las probetas impregnadas se estibaron bajo cubierta para su secado natural.

Las propiedades físicas se determinaron como lo establecen las Normas IRAM N 9544, 9543 y 9532, utilizándose los siguientes materiales: balanza tipo Mettler con capacidad de 160 gramos, con precisión de 0,1 miligramos; calibre con precisión de 0,02 milímetros; estufa de secado con termostato para regular temperaturas de $103{ }^{\circ} \mathrm{C} \pm 2{ }^{\circ} \mathrm{C}$; desecadores y cubetas.

Los ensayos mecánicos de flexión estática y compresión paralela a las fibras, fueron realizados según Norma DIN N ${ }^{\circ}$ 52186. Los demás ensayos de: dureza Janka, corte o cizallamiento paralelo a las fibras, tracción perpendicular a las fibras, clivaje o rajadura, como indica la Norma ASTM N ${ }^{\circ} 143 / 52$. Para ello, se utilizó una máquina universal de ensayos, con capacidad máxima de carga de 10 toneladas.

\section{RESULTADOS Y DISCUSION}

\section{Propiedades físicas y mecánicas de la madera}

Los valores promedios obtenidos en la determinación de las propiedades físicas para cada tratamiento se presentan en la Tabla 1.

No se encontraron diferencias significativas en los promedios de los pesos específicos aparente de las maderas impregnadas con respecto a la no impregnada, Figura 1. Las contracciones tangencial y radial dieron valores menores en las maderas impregnadas pero sin diferencias estadísticamente significativas, coincidiendo con Hartfod (1973), donde dice que las maderas impregnadas con CCA repelen el agua, por la formación de complejos trivalentes del cromo al fijarse en la madera, puede observarse en la Figura 2. 
Tabla 1. Pesos específicos, Contracción total, Coeficiente de contracción y anisotropía de contracción de la madera de Pinus taeda L. no impregnada e impregnada con CCA con retenciones de 5, 10 y $15 \mathrm{~kg} / \mathrm{m}^{3}$.

Table 1. Specific weights, total contraction, retractability coefficient and contraction anisotropy of Pinus taeda L. without and with impregnation wiet retention of 5,10 and $15 \mathrm{~kg} / \mathrm{m}^{3}$.

\begin{tabular}{|c|c|c|c|c|c|c|c|c|c|}
\hline \multirow[b]{2}{*}{ Tratamientos } & \multicolumn{9}{|c|}{ Propiedades físicas } \\
\hline & $\begin{array}{c}\text { P. E. } \\
\left(\mathrm{gr} / \mathrm{cm}^{3}\right)\end{array}$ & $\begin{array}{c}\text { P. S. } \\
(\mathrm{gr} / \mathrm{cm} 3)\end{array}$ & $\begin{array}{c}\text { P. B. } \\
(\mathrm{gr} / \mathrm{cm} 3)\end{array}$ & $\begin{array}{l}\text { CT } \\
(\%)\end{array}$ & $\begin{array}{l}\text { CR } \\
(\%)\end{array}$ & $\begin{array}{l}\text { CA } \\
(\%)\end{array}$ & $\mathrm{CoCT}$ & CoCR & CoAn \\
\hline $\begin{array}{l}\text { Madera sin } \\
\text { impregnar }\end{array}$ & $0,51 \mathrm{a}$ & $0,50 \mathrm{a}$ & $0,43 \mathrm{a}$ & $6,24 \mathrm{a}$ & $3,82 \mathrm{a}$ & $0,48 \mathrm{a}$ & $0,18 \mathrm{a}$ & $0,14 \mathrm{a}$ & $1,63 \mathrm{a}$ \\
\hline $\begin{array}{l}\text { Madera impregnada } \\
\text { con CCA } 5 \mathrm{Kg} / \mathrm{m}^{3}\end{array}$ & $0,49 \mathrm{a}$ & $0,48 \mathrm{a}$ & $0,43 \mathrm{a}$ & $5,90 \mathrm{a}$ & $3,77 \mathrm{a}$ & $0,39 \mathrm{a}$ & $0,17 \mathrm{a}$ & $0,14 \mathrm{a}$ & $1,56 \mathrm{a}$ \\
\hline $\begin{array}{l}\text { Madera impregnada } \\
\text { con CCA } 10 \mathrm{Kg} / \mathrm{m}^{3}\end{array}$ & $0,50 \mathrm{a}$ & $0,49 \mathrm{a}$ & $0,45 \mathrm{a}$ & $5,54 \mathrm{a}$ & $3,54 \mathrm{a}$ & $0,35 \mathrm{a}$ & $0,18 \mathrm{a}$ & $0,13 \mathrm{a}$ & $1,56 \mathrm{a}$ \\
\hline $\begin{array}{l}\text { Madera impregnada } \\
\text { con CCA } 15 \mathrm{Kg} / \mathrm{m}^{3}\end{array}$ & $0,52 \mathrm{a}$ & $0,50 \mathrm{a}$ & $0,46 \mathrm{a}$ & $5,80 \mathrm{a}$ & $3,57 \mathrm{a}$ & $0,41 \mathrm{a}$ & $0,20 \mathrm{a}$ & $0,15 \mathrm{a}$ & $1,62 \mathrm{a}$ \\
\hline
\end{tabular}

Valores medios seguidos de igual letra inicial en cada columna indican que no hay diferencias significativas según el test de Tukey $(\mathrm{p} \leq 0,05)$

PE, PS y $\mathrm{PB}=$ Pesos específicos aparentes estacionado al 12\% de humedad, seco y básico; CT, CR y CA= Contracción tangencial, radial y axial; CoCT y CoCR=Coeficiente de contracción tangencial y radial; CoAn=Coeficiente de anisotropía.

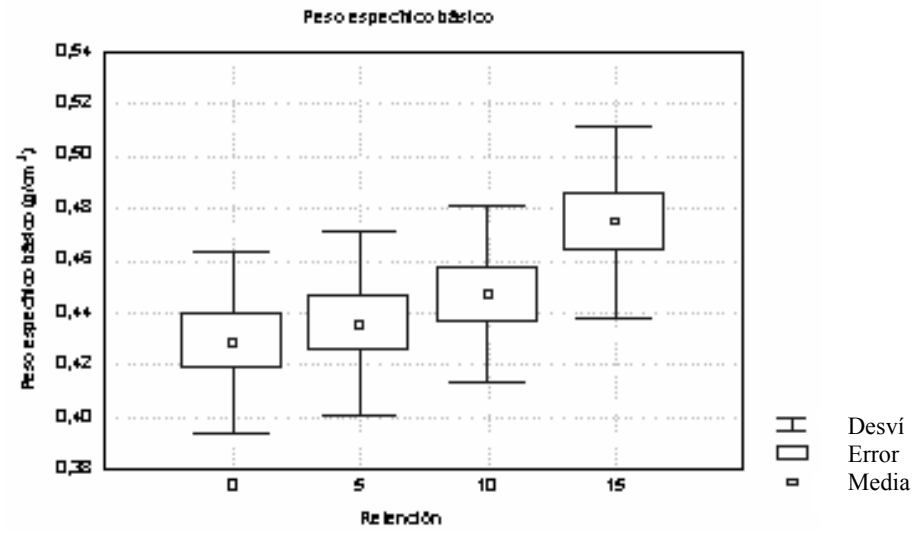

Figura 1. Comparación de medias del peso específico básico de la madera de Pinus taeda L. sin tratar y tratado con 5,10 y $15 \mathrm{~kg} / \mathrm{m}^{3}$.

Figure 1. Comparison of means of basic specific weight of the Pinus taeda L. without and with 5, 10 and $15 \mathrm{~kg} / \mathrm{m}^{3}$.

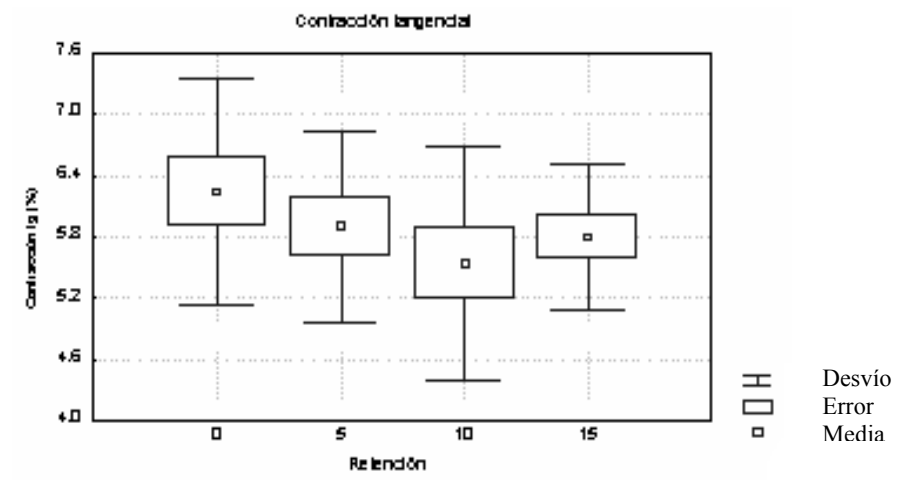

Figura 2. Comparación de medias de contracción tangencial de la madera de Pinus taeda L. sin tratar y tratado con 5,10 y $15 \mathrm{~kg} / \mathrm{m}^{3}$.

Figure 2. Comparison of means of tangential contraction of the Pinus taeda L. without and with 5, 10 and $15 \mathrm{~kg} / \mathrm{m}^{3}$. 
En la Tabla 3 se indican los valores promedios de las propiedades mecánicas de la madera de Pinus taeda sin tratar y tratada con 5,10 y $15 \mathrm{~kg} / \mathrm{m}^{3}$ de retención de CCA.

Los módulos de rotura a la flexión estática en las maderas impregnadas presentaron valores menores respecto a la madera no impregnada, las diferencias no fueron significativas. La madera impregnada con CCA ofrece menor resistencia al módulo de rotura coincidiendo con Pizzi, et al (1984), quiénes manifiestan que las maderas impregnadas pierden tenacidad, ocurre lo contrario con el módulo de elasticidad a la flexión estática, ya que las maderas impregnadas presentaron valores mayores, significando una mayor rigidez debido a la incorporación de metales en la madera. Estas diferencias de valores no fueron estadísticamente significativas.

Los módulos de rotura a la compresión paralela a las fibras presentaron valores menores en sus medias pero sin diferencias significativas (Figura 3). Los módulos de elasticidad a la compresión paralela a las fibras dieron diferencias significativas según el test de Tukey al 0,05 de probabilidad. Las maderas impregnadas con mayores retenciones de CCA, disminuyeron el modulo de elasticidad a la compresión paralela a las fibras, coincidiendo con Wilkinson (1979).

La resistencia al corte tangencial, la resistencia al corte radial, y la resistencia a la tracción en el sentido radial y tangencial no variaron sus valores medios, este resultado se asemeja con lo expuesto por Wilkinson (1979).

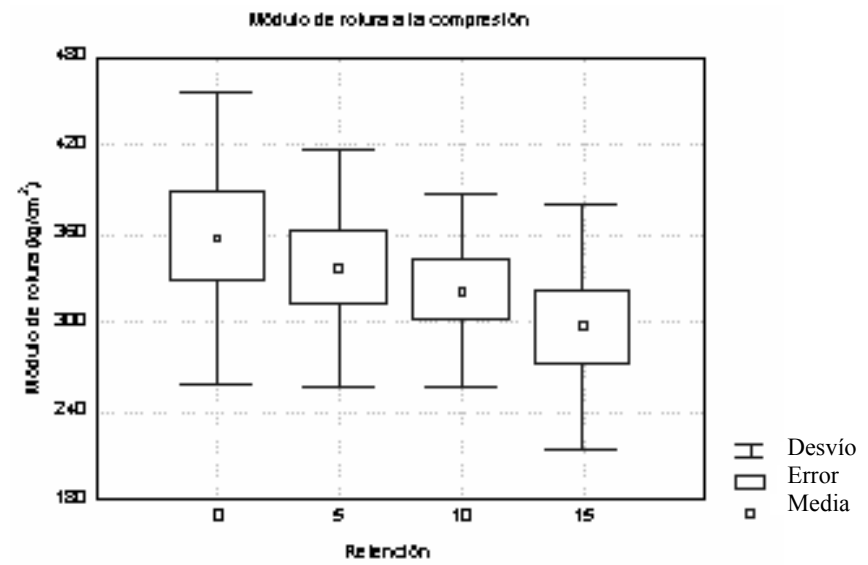

Figura 3. Comparación de medias del módulo de rotura para la compresión paralela a las fibras de la madera de Pinus taeda L. sin tratar y tratada con 5,10 y $15 \mathrm{~kg} / \mathrm{m}^{3}$.

Figure 3. Comparison of means of the breaking module for the parallel compression of the fibers of the Pinus taeda L. timber, nithout and with treatment of 5,10 and $15 \mathrm{~kg} / \mathrm{m}^{3}$. 
Tabla 3. Flexión estática, compresión paralela a las fibras, corte paralelo a las fibras, tracción perpendicular a las fibras, clivaje o rajadura, dureza Janka de la madera de Pinus taeda L. Sin impregnar e impregnada con CCA, con 5,10 y $15 \mathrm{~kg} / \mathrm{m}^{3}$ de retenciones.

Table 3. Static bending, parallel compression to the fibers, parallel cut to the fibers, perpendicular tension to the fibers, cleavage, Janka hardness of Pinus taeda L. without impregnated and impregnated with CCA, with retentions of 5,10 and $15 \mathrm{~kg} / \mathrm{m}^{3}$.

\begin{tabular}{|c|c|c|c|c|c|c|c|c|c|c|c|c|c|}
\hline \multirow{2}{*}{ Tratamientos } & \multicolumn{13}{|c|}{ Propiedades mecánicas } \\
\hline & $\begin{array}{c}\text { MR-F } \\
\left(\mathrm{Kg} / \mathrm{cm}^{2}\right)\end{array}$ & $\begin{array}{c}\mathrm{ME}-\mathrm{F} \\
\left(\mathrm{Kg} / \mathrm{cm}^{2}\right)\end{array}$ & $\begin{array}{c}\text { MR-C } \\
\left(\mathrm{Kg} / \mathrm{cm}^{2}\right)\end{array}$ & $\begin{array}{c}\mathrm{ME}-\mathrm{C} \\
\left(\mathrm{Kg} / \mathrm{cm}^{2}\right)\end{array}$ & $\begin{array}{c}\tau \mathrm{Ctg} \\
\left(\mathrm{Kg} / \mathrm{cm}^{2}\right)\end{array}$ & $\begin{array}{c}\tau \mathrm{Crd} \\
\left(\mathrm{Kg} / \mathrm{cm}^{2}\right)\end{array}$ & $\begin{array}{c}\sigma \mathrm{Ttg} \\
\left(\mathrm{Kg} / \mathrm{cm}^{2}\right)\end{array}$ & $\sigma \operatorname{Trd}\left(\mathrm{Kg} / \mathrm{cm}^{2}\right)$ & $\sigma \operatorname{Rtg}\left(\mathrm{Kg} / \mathrm{cm}^{2}\right)$ & $\begin{array}{c}\sigma \mathrm{Rrd} \\
\left(\mathrm{Kg} / \mathrm{cm}^{2}\right)\end{array}$ & $\operatorname{Dtg}\left(\mathrm{Kg} / \mathrm{cm}^{2}\right)$ & $\operatorname{Drd}\left(\mathrm{Kg} / \mathrm{cm}^{2}\right)$ & Dtrans. $\left(\mathrm{Kg} / \mathrm{cm}^{2}\right)$ \\
\hline Madera sin impregnar & $547,06 \mathrm{a}$ & $39260,54 a$ & $357,62 \mathrm{a}$ & $31360,93 \mathrm{a}$ & $100,36 \mathrm{a}$ & $93,09 \mathrm{a}$ & $35,86 \mathrm{a}$ & $41,19 \mathrm{a}$ & $41,78 \mathrm{a}$ & $48,11 \mathrm{a}$ & $297,25 \mathrm{a}$ & $261,50 \mathrm{a}$ & $299,45 b$ \\
\hline $\begin{array}{l}\text { Madera impregnada } \\
\text { con CCA. } \\
5 \mathrm{Kg} / \mathrm{m}^{3}\end{array}$ & $466,87 \mathrm{a}$ & $32023,72 \mathrm{a}$ & $336,95 \mathrm{a}$ & $24349,38 \mathrm{ab}$ & $105,57 \mathrm{a}$ & $92,29 \mathrm{a}$ & $31,08 \mathrm{a}$ & $38,77 \mathrm{a}$ & $28,44 \mathrm{c}$ & $40,52 \mathrm{a}$ & $294,30 \mathrm{a}$ & $277,90 \mathrm{a}$ & $359,00 \mathrm{~b}$ \\
\hline $\begin{array}{c}\text { Madera impregnada } \\
\text { con CCA. } \\
10 \mathrm{Kg} / \mathrm{m}^{3}\end{array}$ & $474,30 \mathrm{a}$ & $40346,83 a$ & $321,42 \mathrm{a}$ & $19417,64 b$ & $106,74 a$ & $93,89 \mathrm{a}$ & $31,68 \mathrm{a}$ & $43,62 \mathrm{a}$ & $36,42 \mathrm{~b}$ & $45,51 \mathrm{a}$ & $354,10 \mathrm{a}$ & $296,70 \mathrm{a}$ & $462,40 \mathrm{a}$ \\
\hline $\begin{array}{l}\text { Madera impregnada } \\
\text { con CCA. } \\
15 \mathrm{Kg} / \mathrm{m}^{3}\end{array}$ & $468,52 \mathrm{a}$ & $42228,58 \mathrm{a}$ & $297,06 a$ & $18399,40 \mathrm{~b}$ & $104,66 \mathrm{a}$ & $92,39 \mathrm{a}$ & $31,74 a$ & $43,36 \mathrm{a}$ & $37,89 \mathrm{ab}$ & $42,07 \mathrm{a}$ & $325,2 \mathrm{a}$ & $251,00 \mathrm{a}$ & $462,60 \mathrm{a}$ \\
\hline
\end{tabular}

alores medios seguidos de diferente letra inicial en cada columna indican diferencias significativas según el test de Tukey (p $\leq 0,05)$

MR-F y ME-F = Modulo de rotura y modulo de elasticidad a la flexión estática

MR-C y ME-C $=$ Modulo de rotura y modulo de elasticidad a la compresión paralela a las fibras

$\tau \mathrm{Ctg}, \tau \mathrm{Crd}=$ Corte paralelo a las fibras tangencial y radial; $\sigma \mathrm{Ttg}, \sigma \operatorname{Trd}=$ Tracción perpendicular a las fibras tangencial y radial

$\sigma R \operatorname{tg}$,, Rrd= Rajadura o clivage tangencial y radial; Dtg, Drd y Dtrans= Dureza Janka tangencial, radial y transversal 
Las maderas impregnadas presentaron valores mayores que la madera no impregnada en el caso de la dureza en la superficie tangencial y radial sin diferencias significativas, sin embargo presentó diferencia significativa en la superficie transversal, a mayores retenciones de CCA mayor fue la dureza, lo que concuerda con Wilkinson (1979) (Figura 4).

La resistencia al clivaje en el sentido radial y en el sentido tangencial de la madera $\sin$ impregnar, indicaron valores mayores que en la madera impregnada, estadísticamente no fueron significativa en el sentido radial pero si hubo diferencias significativas en el sentido tangencial.

Durera raserersa

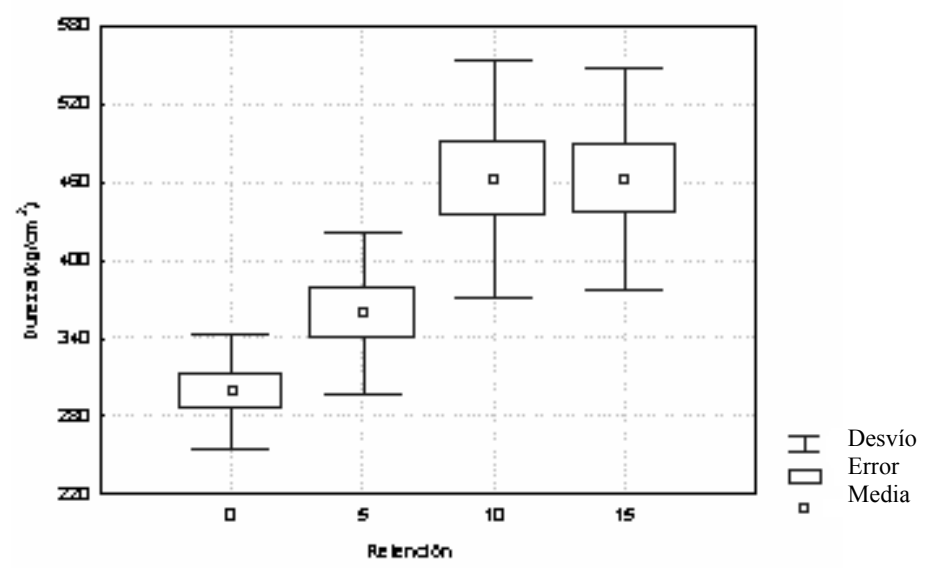

Figura 4. Comparación de medias de dureza Janka transversal de la madera de Pinus taeda L. sin tratar y tratada con 5,10 y $15 \mathrm{~kg} / \mathrm{m}^{3}$.

Figure 4. Comparison of means of transversal Janka hardress of the Pinus taeda L. timber, nithout and with treatment of 5,10 and $1515 \mathrm{~kg} / \mathrm{m}^{3}$.

\section{CONCLUSIONES}

La impregnación con CCA no influye en los pesos específicos aparentes de la madera. Los valores de contracciones en el sentido tangencial, radial y axial en las maderas impregnadas no presentan variaciones estadísticamente significativas.

En cuanto a las propiedades mecánicas: Módulo de rotura, modulo de elasticidad a la flexión estática, módulo de rotura a la compresión paralela a las fibras, tracción perpendicular a las fibras en el sentido radial y en el sentido tangencial, dureza Janka en la superficie radial y en la superficie tangencial, corte paralelo a las fibras radial y tangencial las variaciones en los valores de las medias no son estadísticamente significativas, sin embargo el tratamiento produjo un efecto estadísticamente significativo en la dureza Janka sobre la superficie transversal, Cilvaje en el sentido tangencial, módulo de elasticidad a la compresión paralela a las fibras.

Desde el punto de vista de la construcción la madera del Pinus taeda L. impregnada con CCA con retenciones de 5,10 y $15 \mathrm{~kg} / \mathrm{m}^{3}$ a una presión de $7 \mathrm{~kg} / \mathrm{cm}^{2}$ no es afectada en la mayoría de sus propiedades pero se debe tener en cuentas algunas de las propiedades, en el momento de dimensionar las piezas.

\section{REFERÊNCIAS}

AMERICAN SOCIETY FOR TESTING AND MATERIALS - ASTM. Standard Methods of Small Clear Specimens of Timber. 1997. D 143-94.

AMERICAN WOOD PRESERVERS ASOCIATION - AWPA. Standard P5/86. Standards for waterborne Preservatives. 1996. 
CORONEL, E. O. Fundamentos de las propiedades físicas y mecánicas de las maderas. Primera parte: Fundamentos de las propiedades físicas de la madera. Instituto de tecnología de la madera, Fac. de Cs. Forestales. Santiago del Estero. Argentina. 1994.

DEUTSCHE INDUSTRIE NORM-DIN. N 52186.

FAHLSTROM, G.B. Copfer-Chrome-Arsenate Wood preservatives a study of the influence of composition on service perfomance. Repr. Proceedings of American Wood Preservers Association. p. 6. 1978.

GONZALEZ, R; PEREYRA, O; SUIREZS, T. Propiedades Físicas y Mecánicas de la madera de Pinus elliottii reforestado en la Provincia de Misiones, Argentina. Yvyraretá Año 3 No 3. p.5-19. 1992

HARTFORD, W.H. Chemical and Physical Propierties of wood preservatives and woodpreservatives systems. Wood deterioration and its prevention by preservite treatments. Volumen II, Presrvatives and presrvatives systems. Syracuse University Press. 1973.

INSTITUTO ARGENTINO DE RACIONALIZACIÓN DE MATERIALES-IRAM 9532. Método de determinación de la humedad. 1963.

INSTITUTO ARGENTINO DE RACIONALIZACIÓN DE MATERIALES-IRAM 9543. Método de determinación de las contracciones totales; axial, radial y tangencial y el punto de saturación de las fibras. 1966 .

INSTITUTO ARGENTINO DE RACIONALIZACIÓN DE MATERIALES-IRAM 9544. Método de la determinación de la densidad aparente. 1973.

LEPAGE, E.S. Manual de Preservação de madeiras. v. I. Instituto de Pesquisas Tecnológicas do Estado de Sao Paulo. p.1-7. 1986.

PELAGALLO, A. La impregnación ofrece seguridad para usar madera en la construcción. ASORA $\mathrm{N}^{\circ}$ 11. Año 3. p. 38-40. 1997.

PIZZI, A. et all. Experimental variations in the distribution of CCA preservative in lignin and holocellulose as a function of treating conditions (temperature, concentration, $\mathrm{Ph}$, species and time). Stockholm. The International Research Group on Wood Preservation. Working Group III: Preservatives and methods of treatament. p. 22. 1984.

RAMIREZ, I. G. La preservación contribuye a prolongar la vida útil y el valor de la madera. ASORA N ${ }^{\circ}$. Año 2. p. 28-30. 1996.

SECRETARIA DE AGRICULTURA, GANADERIA, PESCA Y AFINES-SAGPyA. Argentina. Oportunidades de inversión en bosques cultivados. Proyecto Forestal de Desarrollo. Buenos Aires. 1999. p. 77.

TINTO, J.C. Manual para tratamiento protectores de productos forestales. Argentina. Consejo General de Inversiones, 1980.

TUSET, R. Y DURAN, F. Manual de maderas comerciales, equipos y procesos de utilización. Montevideo. Editorial Agropecuaria, Hemisferio Sur S.R.L., 1979.

WILKINSON, J. G. Industrial timber preservation. London. Associated Business Press, 1979. 